\title{
A single-step purification of bothropstoxin-1
}

\section{P.J. Spencer ${ }^{1}$, S.D. Aird" M. Boni-M itake ${ }^{1}$, N. Nascimento ${ }^{1}$ and J.R. Rogero ${ }^{1}$}

\author{
${ }^{1}$ Coordenadoria de Bioengenharia, Supervisão de Radiobiologia, IPEN/CNEN SP, \\ São Paulo, SP, Brasil \\ ${ }^{2}$ Departamento de Biofísica e Radiobiologia, Universidade Federal de Pernambuco, \\ Recife, PE, Brasil
}

\section{Correspondence \\ P.J. Spencer \\ Coordenadoria de Bioengenharia \\ Supervisão de Radiobiologia \\ TBR, IPEN/CNEN SP \\ Travessa R, 400 \\ 05508-900 São Paulo, SP \\ Brasil \\ Fax: 55 (011) 816-9232 \\ E-mail: pspencer@ net.ipen.br \\ Research supported by CAPES and CN Pq. Publication supported by FAPESP.}

Received November 21, 1997 Accepted June 10, 1998

\section{Abstract}

Bothrops venoms are complex mixtures of components with a wide range of biological activities. Among these substances, myotoxins have been investigated by several groups. Bothropstoxin-1 (Bthtx-1) is a phospholipase $\mathrm{A}_{2}$-like basic myotoxin from Bothrops jararacussu. The purification of this component involves two chromatographic steps. Although providing a pure material, the association of these two steps is time consuming and a single-step method using high performance chromatography media would be useful. In the present study, we describe a single-step purification method for Bthtx-1. Bothrops jararacussu venom was dissolved in $1 \mathrm{ml}$ buffer. After centrifugation, the supernatant was injected into a Resource-S cation exchange column connected to an FPLC system and eluted with a linear salt gradient. The complete procedure took $20 \mathrm{~min}$, representing a considerable time gain when compared to a previously described method (Homsi-Brandenburgo MI et al. (1988) Toxicon, 26: 615-627). Bthtx1 purity and identity, assessed by SDS-PAGE and N-terminal sequencing, resulted in a single band with a molecular mass of about 14 $\mathrm{kDa}$ and the expected sequence of the first 5 residues, S-L-F-E-L. Although the amount of protein purified after each run is lower than in the previously described method, we believe that this method may be useful for small-scale purifications.

Bothrops venoms are extremely complex mixtures of toxins, enzymes and peptides, possessing a wide range of biological activities (1). Myotoxins derived from phospholipases $\mathrm{A}_{2}$ have been isolated from venoms of several species $(2,3)$. Some of these are devoid of catalytic activity (2-4). These nonenzymatic myotoxins exhibit high specificity for skeletal muscle and induce severe myonecrosis (2). Characteristically, these toxins display high isoelectric points (above $\mathrm{pH} 8$ )
$(2,3,5,6)$, molecular masses of approximately $13.5 \mathrm{kDa}(5,6)$ and amino acid substitutions in the calcium-binding loop $(4,5)$, which presumably abolishes the calcium-binding capacity. Homsi-Brandenburgo et al. (2) isolated bothropstoxin-1, the main myotoxic component of Bothrops jararacussu venom. Their purification protocol involved gel filtration on Sephadex G-75 followed by cation exchange using SP Sephadex C-25. Since then, other researchers $(5,6)$ have employed 
Figure 1 - lon-exchange chromatography of $25 \mathrm{mg} \mathrm{B}$. jararacussu venom dissolved in $1 \mathrm{ml} 50 \mathrm{mM}$ sodium acetate, $\mathrm{pH}$ 5, on a 1-ml Resource-S column $(6.4 \times 30$ $\mathrm{mm})$, bead size: $15 \mu \mathrm{m}$. A buffer: $25 \mathrm{mM}$ sodium phosphate, $\mathrm{pH}$ 7.8. B buffer: $25 \mathrm{mM}$ sodium phosphate buffer, pH 7.8, containing $2 \mathrm{M} \mathrm{NaCl}$. Flow rate: 2.5 $\mathrm{ml} / \mathrm{min}$. The gradient is indicated by the dashed line. The procedure was carried out at room temperature. similar procedures for the isolation of these non-catalytic myotoxins. Although the above method (2) yields a pure product, it is quite time consuming. In the present study, we describe a single-step purification for bothropstoxin-1.

Bothrops jararacussu venom (25 mg) (Instituto Butantan) was dissolved in $1 \mathrm{ml} 50$ $\mathrm{mM}$ sodium acetate, $\mathrm{pH}$ 5. After centrifugation, the supernatant was injected into a 1-ml Resource-S cation exchange column con-

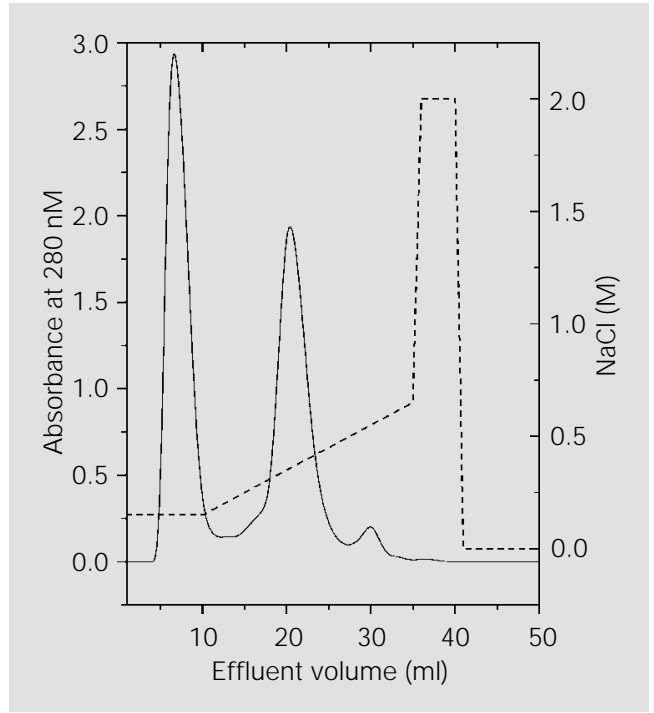

Figure 2 - SDS-PAGE (15\% $100 \mathrm{x}$ $80 \times 0.75 \mathrm{~mm}$ gel) of molecular weight markers (lane 1), crude Bothrops jararacussu venom (lane 2) and purified bothropstoxin-1 (lane 3). Twenty-five $\mu \mathrm{g}$ of each sample, dissolved in 20 $\mu$ sample buffer was applied to each well. The current was fixed at $20 \mathrm{~mA}$ and, following the run, the gel was stained with Coomassie blue R-250.

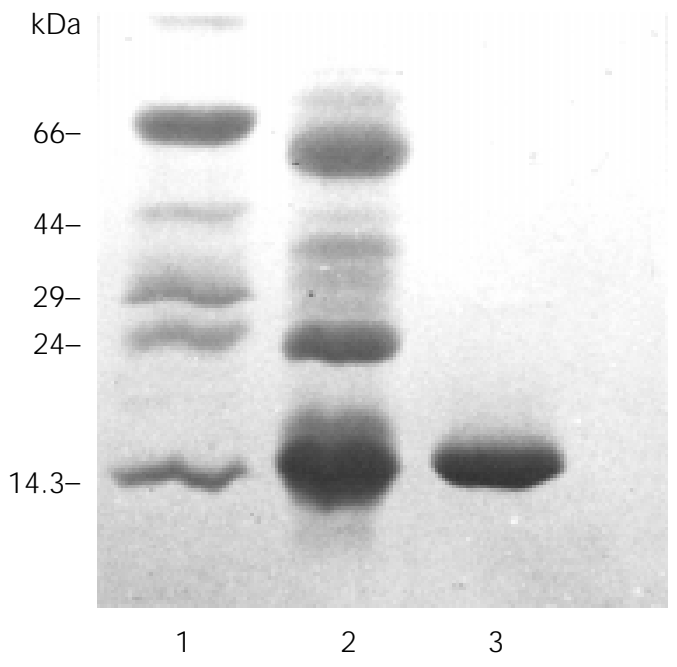

nected to a dual pump FPLC system (Pharmacia Biotech, Uppsala, Sweden). Buffers $\mathrm{A}$ and $\mathrm{B}$ consisted of $25 \mathrm{mM}$ sodium phosphate buffer, $\mathrm{pH} 7.8$, and $25 \mathrm{mM}$ sodium phosphate buffer, $\mathrm{pH} 7.8$, containing 2 $\mathrm{M} \mathrm{NaCl}, \mathrm{pH} 7.8$, respectively. Flow rate was $2.5 \mathrm{ml} / \mathrm{min}$. After an initial 10-ml wash with $7.5 \% \mathrm{~B}$ buffer $(0.15 \mathrm{M} \mathrm{NaCl})$, elution of bound fractions was performed using a linear gradient (slope $=1 \% / \mathrm{ml}$ ) for $25 \mathrm{ml}$. The column was then washed with $10 \mathrm{ml}$ of $\mathrm{B}$ buffer, followed by $10 \mathrm{ml} \mathrm{A}$ buffer to wash $\mathrm{NaCl}$ out of the column.

Aliquots of crude venom, molecular weight standards and purified bothropstoxin1 were submitted to sodium dodecyl sulfate polyacrylamide gel electrophoresis (SDSPAGE) under reducing conditions (7). The purified myotoxin was also submitted to Nterminal sequencing using automated Edman degradation.

The protein was sequenced in an Applied Biosystems 473 A protein sequencer from the Center of Protein Sequencing, Amino Acid Analysis and Peptide Synthesis of the Department of Biochemistry, Institute of Chemistry, University of São Paulo.

Ion-exchange chromatography resulted in three major peaks (Figure 1). The first was eluted before the gradient was applied, the second was eluted in approximately $0.42 \mathrm{M}$ $\mathrm{NaCl}$ and the third in $0.56 \mathrm{M}$ salt. The major peak (peak 2) was dialyzed against deionized water and freeze-dried.

SDS-PAGE (Figure 2) of the purified fraction resulted in a single band with a molecular mass near $14 \mathrm{kDa}$. However, contaminating phospholipases may have co-migrated with bothropstoxin-1. Several proteins with molecular weights ranging from 12.9 to $15.5 \mathrm{kDa}$ have been identified in $B$. jararacussu venom (2), so the appearance of a single band on SDS-PAGE was insufficient to demonstrate purity.

Edman degradation yielded a single se- 
quence, $\mathrm{NH}_{2}$-S-L-F-E-L, which was the same as that reported for bothropstoxin-1 $(2,5)$, indicating that the complete purification of bothropstoxin- 1 was achieved by the method described.

The proposed method reduced purification time by roughly 140 -fold when compared to the method of Homsi-Brandenburgo et al. (2), but dialysis or desalting may be required after purification. Although the amount of protein purified per run is low, due to the small column employed, this method could be readily scaled up for larger amounts of materials.

\section{Acknowledgments}

The authors wish to express their gratitude to André G. Tempone and to Helena Costa for technical assistance.

\section{References}

1. Leite LCC, Furtado MFD, Correa TC \& Raw I (1992). Characterization of the snake venoms from seven Brazilian species of Bothrops by FPLC anion-exchange chromatography. Comparative Biochemistry and Physiology, 102B: 515-520.

2. Homsi-Brandenburgo MI, Queiroz LS, Santos-Neto $H$, Rodrigues-Simioni $L \&$ Giglio J R (1988). Fractionation of Bothrops jararacussu snake venom: partial chemical characterization and biological activity of bothropstoxin. Toxicon, 26: 615-627.

3. Moura da Silva AM, Cardoso DF \&
Tanizaki MM (1990). Differences in distribution of myotoxic proteins in venoms from different Bothrops species. Toxicon, 28: 1293-1301.

4. Francis B, Gutierrez J M, Lomonte B \& Kaiser II (1991). Myotoxin II from Bothrops asper (terciopelo) venom is a Lysine-49 phospholipase $\mathrm{A}_{2}$. Archives of Biochemistry and Biophysics, 284: 352-359.

5. Cintra ACO, Marangoni S, Oliveira B \& Giglio J R (1993). Bothropstoxin-1: amino acid sequence and function. J oumal of Protein Chemistry, 12: 57-64.
6. Mancuso LC, Correa MM, Vieira CA, Cunha OAB, LachattJJ , Selistre de Araujo HS, Ownby CL \& Giglio J R (1995). Fractionation of Bothrops pirajai snake venom: isolation and characterization of piratoxin-1, a new myotoxic protein. Toxicon, 33: 615-626.

7. Laemmli UK (1970). Cleavage of structural proteins during the assembly of the head of bacteriophage T4. Nature, 227: 680-685. 